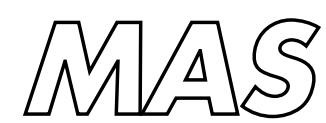

Modelling, Analysis and Simulation

\footnotetext{
Modelling, Analysis and Simulation

The adaptiveness of defence strategies against cuckoo parasitism

R. Planqué, N.F. Britton, N.R. Franks, M.A. Peletier

Report MAS-R0224 September 30, 2002
} 
CWI is the National Research Institute for Mathematics and Computer Science. It is sponsored by the Netherlands Organization for Scientific Research (NWO).

$\mathrm{CWI}$ is a founding member of ERCIM, the European Research Consortium for Informatics and Mathematics.

CWI's research has a theme-oriented structure and is grouped into four clusters. Listed below are the names of the clusters and in parentheses their acronyms.

Probability, Networks and Algorithms (PNA)

Software Engineering (SEN)

Modelling, Analysis and Simulation (MAS)

Information Systems (INS)

Copyright @ 2001, Stichting Centrum voor Wiskunde en Informatica

P.O. Box 94079, 1090 GB Amsterdam (NL)

Kruislaan 413, 1098 SJ Amsterdam (NL)

Telephone +31205929333

Telefax +31205924199

ISSN 1386-3703 


\title{
The Adaptiveness of Defence Strategies Against Cuckoo Parasitism
}

\author{
Robert Planqué ${ }^{\text {, Nicholas F. Britton }}{ }^{2}$, Nigel R. Franks ${ }^{3}$, Mark A. Peletier ${ }^{1}$ \\ ${ }^{1} \mathrm{CWI}$ \\ P.O. Box 94079, 1090 GB Amsterdam, The Netherlands \\ ${ }^{2}$ Department of Mathematical Sciences, University of Bath \\ Bath, BA2 7AY, UK \\ nfb@maths.bath.ac.uk \\ ${ }^{3}$ School of Biological Science, University of Bristol \\ Woodland Road, Bristol BS8 1UG, UK
}

\begin{abstract}
Host bird species of the Eurasian Cuckoo, Cuculus canorus, often display egg-discrimination behaviour but chick-rejection behaviour has never been reported. In this paper, we analyse a host-cuckoo association in which both population dynamics and evolutionary dynamics are explored in a discrete-time model. We introduce four host types, each with their own defence behaviour, displaying either egg or chick rejection, neither or both. We also introduce fitness functions for each of these host types. Although we can characterise the long term behaviour in many cases by a simple heuristic argument which is in accordance with common views in ecology, there are a number of other phenomena that are not explained within this framework: we describe stable oscillatory behaviour and coexistence of two defensive host types. We analyse the scenarios in which chick rejection may establish itself and give a first explanation as to why this defence trait has never been recorded in nature. We find that chick rejectors generally are at an intrinsic disadvantage with respect to a host type that rejects eggs. Hosts benefit more from rejecting cuckoo eggs than cuckoo chicks, and our model suggests that this is chiefly responsible for the absence of chick rejection. Moreover, even though it seems that chick rejection must be useful as an extra defence, it is shown that hosts with both defence strategies are less likely to establish themselves in competition with egg-rejectors than hosts which reject chicks only. These results provide insight in the extent to which adaptations may be perfected by natural selection.
\end{abstract}

2000 Mathematics Subject Classification: 39A11, 92D15, 92D 40

Keywords and Phrases: Avian brood parasitism, host defence, common cuckoo, population dynamics

Note: This work was partially carried out under the CWI Theme MAS1

\section{INTRODUCTION}

Defence strategies against predators or more specifically parasites are ubiquitous and diverse (Futuyma 1979, Gilbert 1980). Given the many marvellous adaptations known in the natural world we may sometimes wonder why a certain obvious strategy has not evolved. One seemingly contradictory situation in defence mechanisms is known from ornithology: although host birds often have great abilities to discriminate against Eurasian Cuckoo eggs, there seems to be a complete lack of defence when it comes to cuckoo chicks. Everyone is familiar with the pictures of a gigantic cuckoo chick nearly devouring its foster parents in their attempt to feed it. Why has the defence strategy of chick rejection not evolved among any of the cuckoo's hosts?

This paper is concerned both with the specific case of cuckoos and hosts and with the general and 
fundamental issue of the perfection of evolutionary adaptation. Is natural selection limited in its ability to favour beneficial adaptations? Consider two adaptations that would each enhance the fitness of a single organism (such as the rejection of cuckoo eggs or cuckoo chicks by a host): Might such adaptations compete with one another so that the lesser one is lost notwithstanding its value even in the presence of the first?

\section{CUCKOO PARASITISM}

In this section, we review some basic natural history of the Eurasian Cuckoo, Cuculus canorus. This is necessary to attack the problem of chick discrimination in cuckoo-host systems. It has been known for centuries that cuckoos do not rear their own young. Instead, they lay their eggs in the nest of a pair of passerine birds, and after the egg is hatched the foster parents raise it. The female cuckoo removes one of the host's eggs and lays a single egg in the nest. Having laid the egg, the female cuckoo abandons the nest and leaves the care to the hosts. The egg usually hatches before the eggs of the host. A few hours after hatching, the hatchling, naked and blind, balances each host egg on its back and ejects it from the nest. Host chicks undergo the same fate and the cuckoo chick becomes the only occupier of the nest. Hence, the parasitised hosts lose all their reproductive success associated with that clutch. The host parents do not intervene in the egg-ejection behaviour of the cuckoo chick. The hosts then rear the single cuckoo chick.

The cuckoo population can be subdivided into so-called gentes. On the whole, each gens has specialised in mimicking the colour, patterns of spots and size of the eggs of the particular host it parasitises (Davies and Brooke 1988). Mimicry is not found, however, in cuckoo chicks when compared with the host's chicks. For instance, in the Reed Warbler Acrocephalus scirpaceus, a well studied host of cuckoos, the chicks are small individuals with a pale yellow gape, whereas cuckoo chicks are much larger and have a red gape. This big difference in appearance in cuckoo and host chicks is not exploited by the host to save it from parasitism. The hosts could benefit in two ways if they would reject a cuckoo chick. First, they would save a lot of energy used to raise the cuckoo chick which might, at least, be used by them to survive the winter months. Secondly, they might be able immediately to start a new clutch.

These observations lead us to the main question under investigation in this paper: why has chick rejection not evolved among any of the cuckoo's hosts?

\section{THE MODEL}

We start by explaining the motivation for our approach. Traditionally, research has focused on egg rejection (see (Winfree 1999) for a short review and (Davies 2000) for a detailed account). Both experimental approaches and modelling efforts efforts have contributed to the understanding of this phenomenon. Because of the complete lack of chick-rejection behaviour by all the cuckoo's host species, it is difficult to set up experiments to test any hypotheses concerning chick rejection. Nevertheless, a number of things have been clarified. It has been shown that some of the cuckoo's hosts accept chicks of other species too (Davies and Brooke 1988, 1989), which contradicts earlier suggestions that the cuckoo chick must manipulate its foster parents (Dawkins and Krebs 1979). However, the cuckoo chick seems to be able to persuade its parents to bring enough food by calling excessively (Davies et al. 1998). The area of the gape is a signal for the parents. It indicates the amount of food that is needed to feed the chicks in the nest. A cuckoo chick, although large, has a smaller gape area than the total gape area of a normal host's clutch. The chick needs the same amount of food as a normal clutch (Brooke and Davies 1989), and hence it has to compensate for this lack of stimulus by calling more than a normal clutch of host chicks. 
We will now develop a model to gain insight into the evolutionary aspects of chick rejection. Lotem (1993) assumed chick rejection to be a trait learned by imprinting upon the chicks in the nest, and therefore concluded that there is a simple explanation for the observed lack of chick rejection: this type of defence strategy is never selectively advantageous if the probability of making a discrimination error is non-zero. However, if we assume that defensive behaviour against cuckoo chicks is an innate trait, we are still at a loss (Rothstein and Robinson 1998). It is this side of the problem we address in this paper.

To motivate the choice of our model we briefly look into previous models for egg rejection. Early models have focused either on population dynamics (May and Robinson 1985) or on population genetics (Rothstein 1975, Kelly 1987, Brooker et al. 1990). Takasu et al. (1993) and Takasu (1998) have been the first to include both ecological and evolutionary aspects of the problem. This more dynamic approach was seen to be necessary following the observation of rapid changes in the defence behaviour by one of the cuckoo's hosts in Japan (Nakamura 1990). Their model has been effective in giving plausible and experimentally testable explanations of two phenomena. First, it has shown that changes in egg-rejection behaviour may be caused by a change in parasitic pressure (Takasu et al. 1993). Second, it has clarified the difference in distribution of egg-rejection behaviour among hosts of the Eurasian Cuckoo versus those of the Brown-headed Cowbird, Molothrus ater, on the basis of their specialist vs. generalist parasitic traits (Takasu 1998). In our attempt to explain the lack of chick rejection we have a slightly more complicated situation than in the case of egg rejection: the latter already exists among many of the cuckoo's hosts. Hence we have to investigate whether chick rejection could invade a population that already exhibits defensive behaviour towards cuckoo eggs. Is the cuckoo-host system exhibiting an evolutionary equilibrium or an evolutionary lag? It is hence necessary to include at least three host types: hosts that accept both eggs and chicks, hosts that reject eggs and hosts that reject chicks. For completeness, we shall also include a host type that rejects both eggs and chicks.

Before we introduce our model we will make some general remarks. As we have seen the cuckoo has many hosts but each gens generally specialises on only one or perhaps two. Therefore, in this paper we focus on one gens and its one host. Contrary to for instance Lotem (1993) we assume that the host defence systems are determined by hereditary factors. Recent studies on defensive behaviour by cuckoo hosts support this assumption (Rothstein 2000). We treat the various host types as separate species of birds. We combine a clonal model for the four host types with two predator-prey equations for the interaction between cuckoos and hosts to investigate the brood parasite system. It is widely assumed that there are costs associated with displaying egg rejection, due to discrimination errors (May and Robinson 1985, Davies and Brooke 1988, Rohwer et al. 1989, Røskaft et al. 1990, Rothstein 1990, Moksnes et al. 1991, Marchetti 1992). These egg-rejection costs are assumed to be small but are taken into account in our analysis, and will play an essential role. In this paper we assume that chickrejection behaviour also entails similar recognition costs. We do not take into account any hereditary variation within the cuckoo population.

Let $P_{t}$ be the population density of female cuckoos and $H_{t}$ that of the female hosts in year $t$. We assume that surviving offspring breed in the year after they are hatched. If the female cuckoo finds a nest she will lay a single egg and the chick will grow up with a constant probability $G$ to survive to the next breeding season. The adult female cuckoo survives to the next season with a probability $s_{P}$. Similarly, we introduce a constant $s_{H}$ which measures the intrinsic survival rate of adult host birds. Here, as in the rest of the paper, we neglect any effects due to intra-specific competition.

We distinguish four host types: all-acceptors, egg-rejectors, chick-rejectors and all-rejectors. Their frequencies in the total host population are denoted by $h_{t}^{a}, h_{t}^{e}, h_{t}^{c}, h_{t}^{e c}$ respectively, which add up to one. 
The cuckoo is assumed to perform a random search with a search efficiency measured by a parameter $a$, called the area of discovery by Nicholson and Bailey (1935). The probability that a host nest escapes from parasitism is thus given by $e^{-a P_{t}}$, the zeroth term in a Poisson distribution (May and Robinson 1985). The density of cuckoos in the next generation is

$$
P_{t+1}=s_{P} P_{t}+\left(1-e^{-a P_{t}}\right) H_{t} G\left(h_{t}^{a}+q_{e} h_{t}^{e}+q_{c} h_{t}^{c}+q_{e c} h_{t}^{e c}\right)
$$

see also (Takasu et al. 1993). The first term corresponds to the surviving adults, the second to the successfully raised young from the nests that have not escaped parasitism. We will introduce the constants $q_{e}, q_{e}$, and $q_{e c}$ in a moment, and explain their occurrence in equation (1) at the end of the section.

The total density of offspring in the host population is a sum over the contributions from the different host types:

$$
H_{t}\left(f_{a} h_{t}^{a}+f_{e} h_{t}^{e}+f_{c} h_{t}^{c}+f_{e c} h_{t}^{e c}\right) .
$$

Here we have introduced fitness functions for each of the host types, denoted by $f_{a}, f_{e}, f_{c}$, and $f_{e c}$. They will be discussed shortly. In the absence of cuckoo parasitism the number of individuals in a certain area is limited by the available resources. Taking this factor into account with a parameter $k$, the host density for the next year is given by

$$
H_{t+1}=\frac{H_{t}}{1+\frac{H_{t}}{k}}\left(s_{H}+f_{a} h_{t}^{a}+f_{e} h_{t}^{e}+f_{c} h_{t}^{c}+f_{e c} h_{t}^{e c}\right) .
$$

We will now discuss the fitness functions of the host types. In general all host types will suffer to some extent from an increase in parasitism by the cuckoos, in the sense that they will lose some offspring. So for all host types we assume that the fitness functions are monotonically decreasing functions of $P_{t}$. In the absence of parasitism however, we expect some differences in the number of offspring produced by the various host types. Since we have assumed that the rejecting host types make some errors in their attempts to discriminate cuckoo eggs or chicks, we assume that the all-accepting pairs have a slight advantage when the parasitic pressure is low.

Now let $f$ be the number of offspring per annum raised by an all-accepting host pair that is not parasitised. If we multiply this by the probability for such a pair to escape parasitism we find the number of offspring for all-acceptor pairs in terms of cuckoo density:

$$
f_{a}=f e^{-a P_{t}}
$$

The corresponding fitness functions for the rejector types are given by

$$
\begin{aligned}
& f_{e}=e_{1} f e^{-a P_{t}}+e_{2} f\left(1-e^{-a P_{t}}\right), \\
& f_{c}=c_{1} f e^{-a P_{t}}+c_{2} f\left(1-e^{-a P_{t}}\right), \\
& f_{k}=k_{1} f e^{-a P_{t}}+k_{2} f\left(1-e^{-a P_{t}}\right),
\end{aligned}
$$

where $e_{1} f$ and $e_{2} f$ are the expected number of offspring per annum raised by unparasitised and parasitised egg-rejectors respectively, and similarly for chick-rejectors and all-rejectors. 
We shall neglect any physiological costs associated with the behavioural capability for rejection, but shall take account of the costs of recognition errors of types I and II. By type I errors we mean mistakenly ejecting one's own egg or chick in the absence of cuckoo parasitism. Let $p_{e}$ be the probability that an egg rejector makes a type I error and removes one of its own eggs by mistake, and $b_{e}$ the relative pay-off for raising a clutch with one egg removed. Then

$$
e_{1}=\left(1-p_{e}\right)+p_{e} b_{e} .
$$

Similarly,

$$
c_{1}=\left(1-p_{c}\right)+p_{c} b_{c},
$$

and

$$
k_{1}=\left(1-p_{e}\right)\left(1-p_{c}\right)+b_{e} p_{e}\left(1-p_{c}\right)+b_{c} p_{c}\left(1-p_{e}\right)+b_{e c} p_{e} p_{c},
$$

with the obvious notation.

By type II errors we mean that the hosts sometimes fail to spot the cuckoo's eggs or chicks in their nests. Let $q_{e}$ be the probability of an egg-rejector making a type II error, i.e. mistakenly accepting a cuckoo egg. The relative pay-off for a host that accepts a cuckoo egg is zero. Hence we have

$$
e_{2}=\left(1-q_{e}\right) b_{e}
$$

We have assumed here that an egg-rejector has the same pay-off $b_{e}$ for rejecting one of its own eggs when unparasitised as it does for rejecting a cuckoo egg when parasitised: it merely has one egg less, in the first case removed by itself and in the second by the laying cuckoo. For chick-rejectors this is not the case: when an unparasitised chick-rejector makes a type I error and ejects one of its own chicks, it still has the rest of its clutch, and its pay-off is $b_{c}$. When it is parasitised and ejects the cuckoo chick, however, its pay-off varies between $b_{e}$ (loss of the egg removed by the laying cuckoo) and zero (loss of the clutch), depending on the damage done by the cuckoo chick before it is discovered and ejected. We shall set the pay-off to $\gamma b_{e}$, where $\gamma$ is a measure of how much of the clutch is saved on average. Thus

$$
c_{2}=\left(1-q_{c}\right) \gamma b_{e} .
$$

For all-rejectors we find

$$
k_{2}=\left(1-q_{e}\right) b_{e}+q_{e}\left(1-q_{c}\right) \gamma b_{e} .
$$

Now note that $0<b_{e c}<b_{c}, b_{e}<1$; it is better to lose either an egg or a chick than to lose both, but it is better still to lose neither. Moreover $b_{c} \leq b_{e}$; if one potential offspring is to be lost it might as well be lost early (at the egg stage), so that it no longer requires resources (however minimal). Note also that $0 \leq p_{e}, p_{c}, q_{e}, q_{c}<1$, with e.g. $p_{e}=0$ if and only if the egg-rejector never makes a type I error. It follows immediately that

$$
0<e_{2}<e_{1} \leq 1, \quad 0<c_{2}<c_{1} \leq 1, \quad 0<k_{2}<k_{1} \leq 1 ;
$$

(i) even rejectors are disadvantaged by parasitism, because the cuckoo ejects one of their eggs when laying its own, and (ii) rejectors are no better off than acceptors in the absence of parasitism (and are worse off unless they never make a type I error). It also follows immediately that

$$
k_{1} \leq e_{1}, c_{1}, \quad k_{2}>e_{2}, c_{2}
$$


(iii) rejectors with both defence strategies are no better off than those with only one in the absence of parasitism (and are worse off unless they never make a type I error), but (iv) they are better off than those with only one if they are parasitised. However, we never see hosts that employ both defence strategies. The explanation of this depends on a subtler argument that we give later. All these inequalities, and hence conclusions (i) to (iv), hold for any allowable parameter values. The relationships between $e_{1}, c_{1}, e_{2}$ and $c_{2}$, on the other hand, depend on the particular parameter values chosen, and in particular on the value of $\gamma$. Unless the biologically unreasonable assumption is made that the cuckoo chick is always discovered before it does any damage then $\gamma<1$. This will play an important part in our explanation of why egg-rejectors rather than chick-rejectors are observed in nature.

An illustration of a typical set of fitness functions can be found in figure 1.

With these fitness functions we may determine the host type frequencies in the next generation:

$$
\begin{aligned}
h_{t+1}^{a} & =h_{t}^{a} \frac{s_{H}+f_{a}}{D}, \\
h_{t+1}^{e} & =h_{t}^{e} \frac{s_{H}+f_{e}}{D} \\
h_{t+1}^{c} & =h_{t}^{c} \frac{s_{H}+f_{c}}{D} \\
h_{t+1}^{e c} & =h_{t}^{e c} \frac{s_{H}+f_{e c}}{D},
\end{aligned}
$$

where $D=s_{H}+f_{a} h_{t}^{a}+f_{e} h_{t}^{e}+f_{c} h_{t}^{c}+f_{e c} h_{t}^{e c}$.

We may now explain the cuckoo equation (1) completely: the defensive host types contribute to the next generation of cuckoos if they have failed to discriminate the cuckoo's eggs or chicks. This amounts to the factors $q_{e}, q_{c}$ and $q_{e c}$ found in the equation.

As a remark, note that $a$ can be scaled out of the equations, but this is not done for two reasons: it reduces the number of parameters only by one giving only a small gain, and the current parameter has a well-defined biological interpretation, contrary to its rescaled counterpart.

Equations (1), (2), and (3) to (6) constitute our model.

ANALYsis

As an introduction to the characteristics of the model we will present a simple, intuitive analysis of a general cuckoo-host system. We believe that this intuitive analysis is helpful, at this stage, even though the full model exhibits behaviour that is both different and more complex than that suggested by the simple intuitive argument. Moreover, the simple argument does include the most important clue to answering the main question discussed in this paper. We will therefore treat it as a background against which we present the more detailed analysis below.

At low cuckoo densities the all-accepting hosts have highest fitness, whereas the defending host types are fitter for high cuckoo densities. Hence, if the cuckoo density were to remain low (or high), the frequency of the accepting (defending) host type will approach unity. Let us assume that the system will converge to an equilibrium solution, and assume the following two dynamical properties of the model:

1. If there are only accepting hosts and the cuckoo numbers are low, the cuckoo numbers 


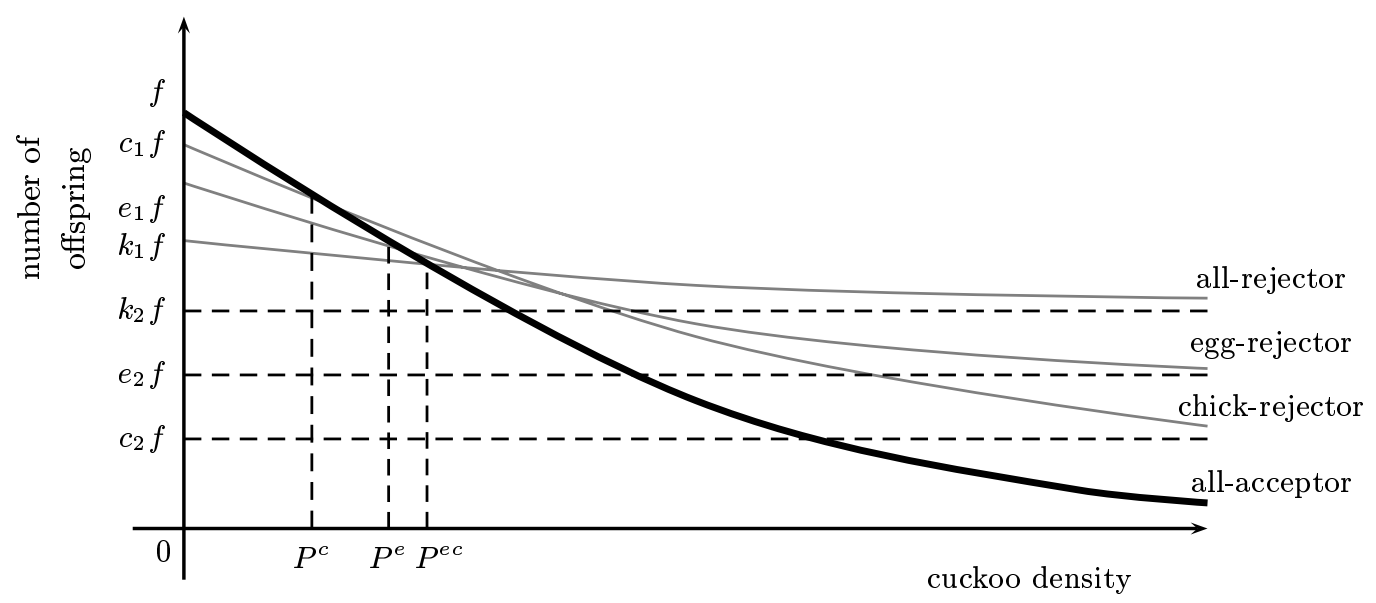

Figure 1: The average number of female offspring for each of the host types, all-acceptor, egg-rejector, chick-rejector and all-rejector pairs, respectively. The three values $P^{e}, P^{c}$ and $P^{e c}$ are the cuckoo densities at which the fitness of the all-acceptor hosts equals the fitness of egg-, chick- and all-rejector hosts respectively. In the diagram they satisfy $P^{c}<P^{e}<P^{e c}$, but this order depends on the parameters of the problem. When $P_{t}$ is small (less than $P^{c}$ ) the acceptor pairs produce more offspring, but for higher cuckoo densities the defending hosts are reproductively fitter than acceptors. Note that the discrimination costs for all-rejectors is proportionally higher than for either egg or chick rejection, but that these hosts are fitter under high parasitic pressure. The dashed horizontal lines are the asymptotic values for each of the defensive fitness functions for large $P_{t}$.

will increase.

2. If there are only defending hosts and the cuckoo numbers are high, the cuckoo numbers will decrease.

Then we expect that there is an equilibrium value for the cuckoo density at which the fitnesses of defending and accepting hosts are equal.

Numerical investigation shows that this argument gives a rough description of the characteristics of the model. We give an illustration of the effect described in the argument in figure 2. However, this argument misses additional behaviour: we may encounter for instance quasi-periodic solutions, or stable coexistence of two defensive host type. We start with a discussion of the solutions described by the heuristic argument.

\section{Intermediate rejection frequencies}

In certain parameter value ranges we find one of three equilibrium solutions which we denote by $S^{e}, S^{c}$ and $S^{e c}$. At $S^{e}$ for instance, we find a steady state with coexistence of all-acceptor hosts and egg-rejectors, i.e. with $h^{a}+h^{e}=1$ and both frequencies positive. Analogous descriptions can be given for the other two solutions $S^{c}$ and $S^{e c}$. The explicit analytic derivation of these solutions is given in the Appendix. Here we merely state that they are of the form

$$
\begin{aligned}
S^{e} & =\left(P^{e}, H^{e}, h^{a}, h^{e}, 0,0\right), \\
S^{c} & =\left(P^{c}, H^{c}, h^{a}, 0, h^{c}, 0\right), \\
S^{e c} & =\left(P^{e c}, H^{e c}, h^{a}, 0,0, h^{e c}\right),
\end{aligned}
$$



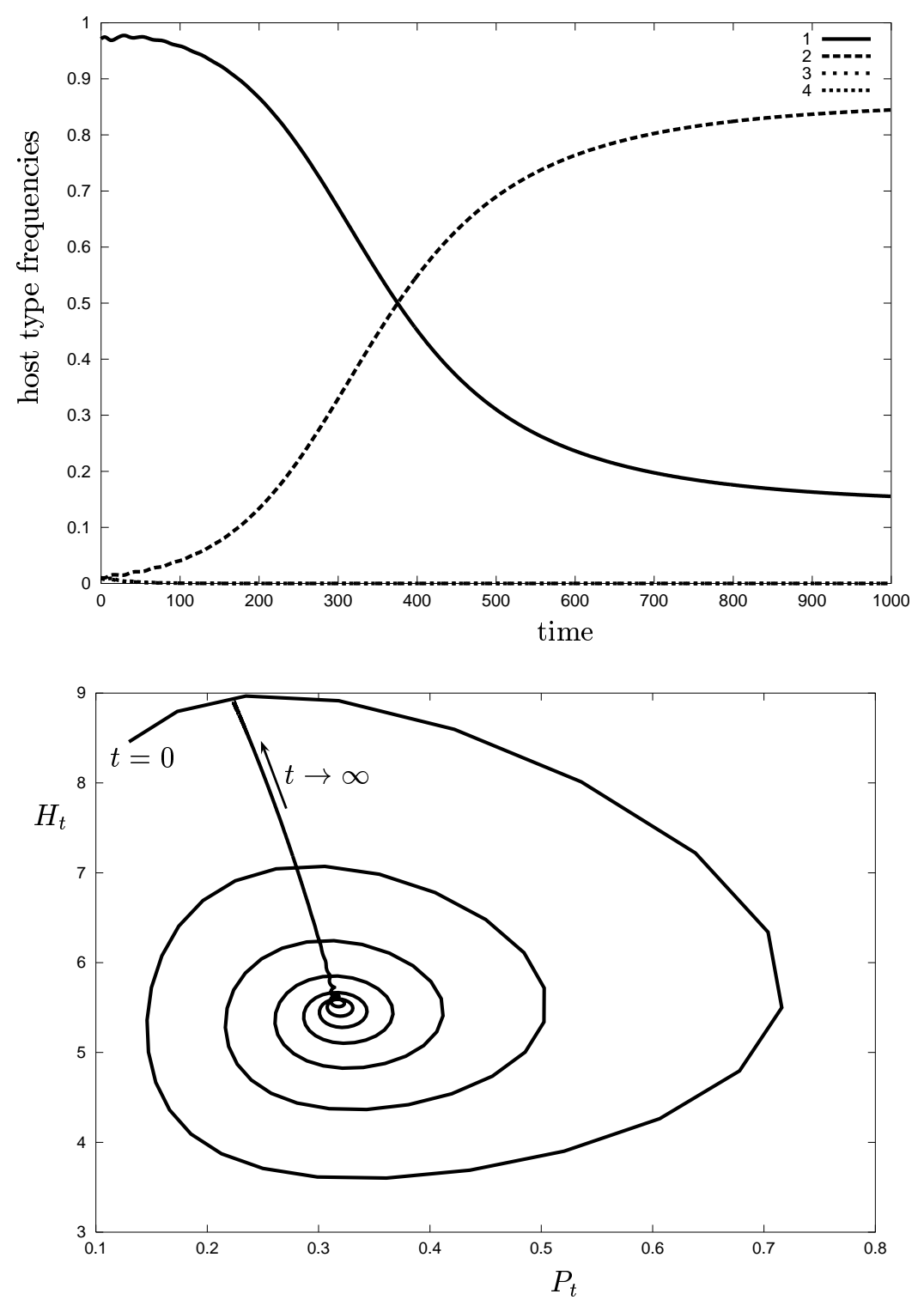

Figure 2: A typical numerical simulation of the model, illustrating damped oscillations converging to a steady state in which egg-rejecting (curve 2) and all-accepting hosts (curve 1) coexist. This equilibrium is called $S^{e}$. Note that chick- rejectors (curve 3) and all-rejectors (curve 4) increase very briefly but are 'outcompeted' by the fitter egg-rejecting hosts and drop to zero almost immediately. The bottom picture shows the temporal dynamics of cuckoos (curve 5) with respect to hosts (curve 6). Parameters used are $s_{H}=0.5, s_{P}=0.5, f=0.7, a=0.7, G=0.15, k=90, e_{1}=0.95, e_{2}=0.3$, $q_{e}=.5, c_{1}=0.9, c_{2}=0.15, q_{c}=.5, k_{1}=0.85, k_{2}=0.375, q_{e c}=.75$ and initial conditions $P_{0}=0.1$, $H_{0}=8, h_{0}^{a}=0.97, h_{0}^{e}=h_{0}^{c}=h_{0}^{e c}=0.01$. In all numerical investigations, we have only changed $s_{H}$, $f, k$, and the discrimination costs. The parameter values conform to those of Takasu et al. (1993). 
where $P^{e}$ denotes the steady state value of $P_{t}$ in the case of intermediate egg rejection, etcetera. The equilibrium values for $P_{t}$ can be determined directly from the fitness functions: at each of these solutions the fitness of all-acceptors is equal to the fitness of the respective defensive host type. So in the case of egg-rejectors, we solve $f_{a}=f_{e}$ for $P_{t}$ to find the desired result.

This result raises the question, to which of these solutions will the system converge? Numerical investigations have shown that there is a simple rule to determine this: one computes the equilibrium cuckoo densities $P^{e}, P^{c}$ and $P^{e c}$ and determines which is the smallest. The equilibrium solution corresponding to this cuckoo density then is the one to which the system will converge. This is a direct application of the heuristic argument stated at the beginning of this section, given that the fitness functions are monotonically decreasing functions in $P_{t}$ : for $P_{t}$ less than the smallest equilibrium value, $P_{t}$ will increase; for $P_{t}$ larger than the smallest equilibrium value, the all-acceptors will have highest fitness and will cause a decrease of $P_{t}$.

We have assumed a number of things in the preceding discussion, any of which may under certain conditions be violated and give additional behaviour not explained by the argument above:

- The system will actually converge to an equilibrium solution.

- In such instances $S^{e}, S^{c}$, or $S^{e c}$ are the only possible steady states.

- Among $P^{e}, P^{c}$, and $P^{e c}$ there is one and only one value which is strictly smaller than the other two.

Each of these assumptions does not have to hold, giving qualitatively different behaviour. These phenomena will now be explored in more detail.

\section{Extending the three equilibria}

The three equilibria $S^{e}, S^{c}$ and $S^{e c}$ may be extended in a natural way to include limit cases. We treat these extensions as separate equilibria since these extensions are not described by the heuristic argument, they are treated as separate equilibria. We describe the occurrence of the following cases: absence of cuckoos, a cuckoo population in coexistence with a completely all-accepting host population, and a cuckoo population in coexistence with a host population that consists of a single defensive host type.

We start by looking at the system in the case of all-acceptance of the host pairs. In general the system will converge to a unique equilibrium solution. The equilibrium solution for the host population in absence of the cuckoo may be found by setting $H_{t+1}=H_{t}$ and $P_{t}=0$ in (2). Let us call this steady state $S^{0}=\left(P^{0}, H^{0}, h^{a}, h^{e}, h^{c}, h^{e c}\right)=\left(0, H^{0}, 1,0,0,0\right)$. We then find

$$
H^{0}=k\left(s_{H}+f-1\right) .
$$

This number corresponds to the carrying capacity of the host population. If $H^{0}<0$ the host population goes extinct since the death rate then exceeds the birth rate $\left(1-f>s_{H}\right)$. From now we will assume that $1-f<s_{H}$.

For larger values of $k$ we find that in the absence of any rejector hosts the system evolves towards an equilibrium solution $S^{1}=\left(P^{1}, H^{1}, 1,0,0,0\right)$, where $P^{1}$ and $H^{1}$ are the unique solution of

$$
\left\{\begin{array}{l}
H^{1}=\frac{\left(1-s_{P}\right) P^{1}}{G\left(1-e^{-a P^{1}}\right)} \\
H^{1}=k\left(f e^{-a P^{1}}+s_{H}-1\right) .
\end{array}\right.
$$


If $P^{1}$ approaches zero we find

$$
\frac{s_{P}-1}{G a}+k\left(s_{H}+f-1\right)=0 .
$$

Therefore the a critical value of our chosen bifurcation parameter $k$ for the survival of the cuckoo population is given by

$$
k^{P}:=\frac{1-s_{P}}{a G\left(s_{H}+f-1\right)} .
$$

For smaller values of $k$ the cuckoo population goes extinct. This argument is identical to the analysis in Takasu et al. (1993).

We now consider the relationship between these two new steady states and $S^{e}, S^{c}$, and $S^{e c}$. Since $h^{e} \in(0,1)$ we may solve $h^{e}>0$ from the analytic expression in (14) in terms of $k$ to find a minimal value for $k$ for $S^{e}$ to be meaningful. Let us call this value $k_{0}^{e}$. Similarly we find minimal values $k_{0}^{c}$ and $k_{0}^{e c}$. The methods to derive these expressions are given in the Appendix. We note here that $k^{P}<k_{0}^{e}, k_{0}^{c}, k_{0}^{e c}$, thus excluding the possibility that any defensive host types could establish themselves before the cuckoos were present. When $k$ is in the interval $\left(k^{P}, \min \left\{k_{0}^{e}, k_{0}^{c}, k_{0}^{e c}\right\}\right)$ the system converges to $S^{1}$.

Intuitively the occurrence of these values $k_{0}^{e}, k_{0}^{c}$ and $k_{0}^{e c}$ can be explained by our expectancy that the cuckoo has to search well enough, and the environmental carrying capacity for the hosts has to be sufficiently high.

We can, on the other hand, also find maximal values for $k$ for these equilibria $S^{e}, S^{c}$ and $S^{e c}$ to be converged upon. This can be done by solving for instance $h^{e}<1$ in the case of $S^{e}$. We refer to the Appendix for the formal calculations. We denote these values by $k_{1}^{e}, k_{1}^{c}$ and $k_{1}^{e c}$. We can now formulate more precisely when one of the three equilibria may be converged upon, which we illustrate again in the case of $S^{e}: S^{e}$ may be attained if $k \in\left(k_{0}^{e}, k_{1}^{e}\right)$ and $P^{e}$ is the smallest of the three equilibrium values $P^{e}, P^{c}$ and $P^{e c}$. Completely analogous conditions can be given for $S^{c}$ and $S^{e c}$.

We thus find natural extensions for all $k>0$ of the equilibria $S^{e}, S^{c}$ and $S^{e c}$. As $k$ increases we go through four stages:

- Only hosts and no cuckoos, $k \in\left(0, k^{P}\right)$.

- Coexistence of cuckoos with all-accepting hosts, $k \in\left(k^{P}, k^{*}\right)$, where $k^{*}=\min \left\{k_{0}^{e}, k_{0}^{c}, k_{0}^{e c}\right\}$.

- Coexistence of cuckoos with a stable mixed population of all-accepting and one defensive host type, $k \in\left(k^{*}, \hat{k}\right)$, where $\hat{k}$ is the corresponding $k_{1}^{e}, k_{1}^{c}$ or $k_{1}^{e c}$.

- Coexistence of cuckoos with a host population existing only of hosts of one defensive type, $k \in(\hat{k}, \infty)$, where $\hat{k}$ is one of $\left\{k_{1}^{e}, k_{1}^{c}, k_{1}^{e c}\right\}$.

This is illustrated in figure 3 in the case of egg-rejectors.

Here we have still assumed two things: the system converges to an equilibrium and the steady state in which one defensive type is the only host type is stable for all $k>\hat{k}$ where $\hat{k}$ is one of $\left\{k_{1}^{e}, k_{1}^{c}, k_{1}^{e c}\right\}$. The violation of the latter condition is discussed later. When the first assumption is not valid we can find quasi-periodic solutions. These are discussed in the next section. 


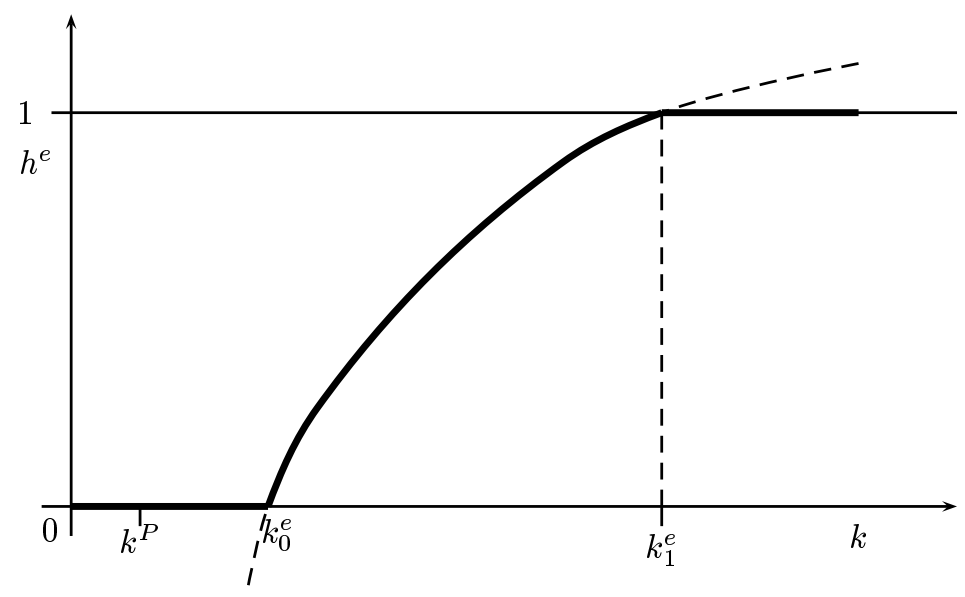

Figure 3: The coexistence of egg-rejectors and all-acceptors at steady state $S^{e}$ (bold curved line, and dashed its hypothetical extension below $h^{e}=0$ and above $h^{e}=1$ ). The extension of $S^{e}$ to all $k>0$ is indicated by the horizontal bold lines. Starting at $k=0$ we find no cuckoos present until $k^{P}$, cuckoos but no defensive hosts between $k^{P}$ and $k_{0}^{e}$, cuckoos and a mixed defensive and accepting host population between $k_{0}^{e}$ and $k_{1}^{e}$ and cuckoos and only egg-rejectors for $k>k_{1}^{e}$. This figure corresponds to curve (1) of figure 4 .

No convergence to an equilibrium solution

Even when the conditions for any of the three steady states is satisfied, the system does not have to converge to any of the three. We again illustrate this in the case of $S^{e}$. There is an asymptotic value for $s_{H}, s_{H}^{e}$ say, with the property that for $s_{H}<s_{H}^{e}$ the steady state $S^{e}$ is not attained. The analytical derivation of $s_{H}^{e}$ is given in the Appendix. This is illustrated in figure 4. Numerical investigations show that there is an $s_{H}$-interval in which $S^{e}$ is not attained, but the system converges to a quasi-periodic orbit (see figure 5 for an example). These stable oscillations are both in cuckoo and host numbers, and also in host type frequencies. Mathematically their appearance corresponds to a Naimark-Sacker bifurcation, the equivalent of the Hopf bifurcation for discrete time systems (see e.g. (Arrowsmith and Place 1990, p. 261)). In figure 4 the occurrence of this bifurcation has been placed in a broader context of parameters. The occurrence of this phenomenon seems to be independent of the stability or occurrence of the three equilibria $S^{e}, S^{c}$ and $S^{e c}$. The $s_{H}$-interval in which these periodic solutions are found is concentrated around the asymptote $s_{H}=s_{H}^{e}$. Note that we also find non-trivial defensive hosts (i.e. $h_{t}^{e}>0$ ) for $s_{H}<s_{H}^{e}$, which do not correspond to the intermediate frequency $h^{e}$ in steady state $S^{e}$. This is not predicted by the heuristic argument.

More coexisting host types

The heuristic argument predicts that the system converges to a steady state in which the cuckoo population is equal to the smallest value of $P^{e}, P^{c}$ and $P^{e c}$. However, for some parameter values we may find a stable coexistence between two defensive host types rather than between one accepting and one defensive host type. As an example, in figure 6 we see that although for smaller values of $k$ we find the familiar coexistence between all-acceptors and egg-rejectors, for larger values of $k$ the egg-rejectors and all-rejectors are in coexistence. This steady state of intermediate egg- and all-rejectors will be 


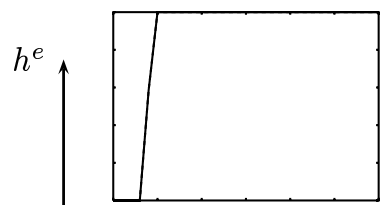

$(1)$

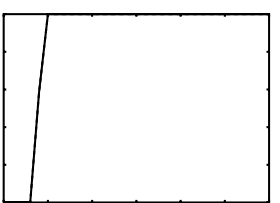

$(2)$

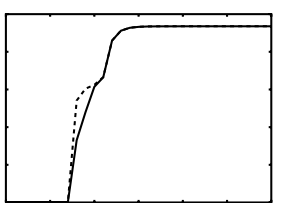

(3)

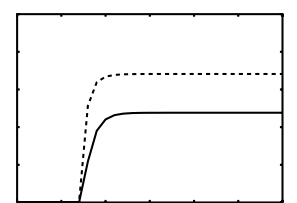

(4)

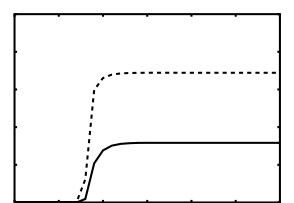

$(5)$

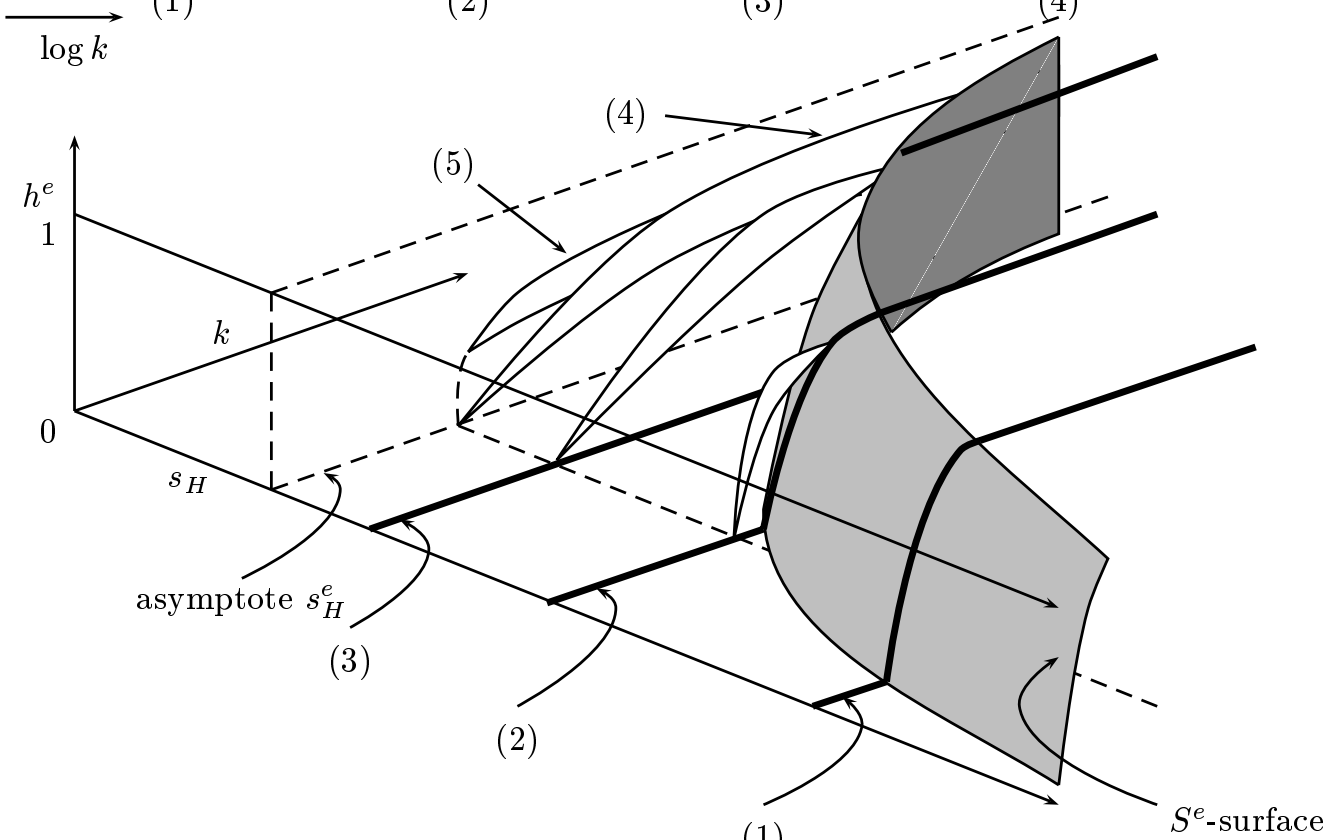

(1)

Figure 4: Assuming $P^{e}$ to be the smallest of $\left\{P^{e}, P^{c}, P^{e c}\right\}$, we find qualitatively different solutions if we vary parameters $k$ and $s_{H}$. With $s_{H}$ large, we find the standard solution $\overline{S^{e}}$, such as (1), (2) and (3). The region with intermediate frequencies, denoted by $S^{e}$ forms a surface in the $\left(k, s_{H}, h^{e}\right)$-space, which asymptotically converges to $k=0$ and $s_{H}^{e}=1-f e_{2} /\left(1-e_{1}+e_{2}\right)$ in the two planes $h^{e}=0$ and $h^{e}=1$. For small values of $s_{H}$ we find quasi-periodic solutions which are confined to the white curved regions, originating at the dashed line. One implication is that solutions exist for $s_{H}$ less than the asymptotic value in which there is sustained egg rejection, for instance (5). The width of the oscillating regions is largest in the $\left(s_{H}, h^{e}\right)$-plane at the $s_{H}$-asymptote, see (4). Keep in mind that for large values of $k, \bar{S}$ e is only locally stable. Keeping initial conditions fixed, the system can make a transition from egg rejection as steady state to all-rejection, as explained in figure 6 . This effect is not shown in the above picture. The five small pictures correspond to the solutions shown in the large picture. Note that in all but the left figure, we find regions where Naimark-Sacker bifurcations have occurred. As in the large picture, figure 1 does not include the instability of the $h^{e}=1$ solution for larger $k$. Figures 2 and 3 do not show the unstable part of the egg-rejector equilibria, namely the part between $h^{e}=0$ and where it attaches to the closed oscillatory regions. These figures have been made with parameter values as in figure 2 but with, from left to right, $s_{H}=0.5,0.375,0.37,0.3768$ and $0.365 ; \log k \in(3.8,8.6)$ (figure 1$)$ and $\log k \in(3.5,18.5)$ (figures 2 to 5 ). 

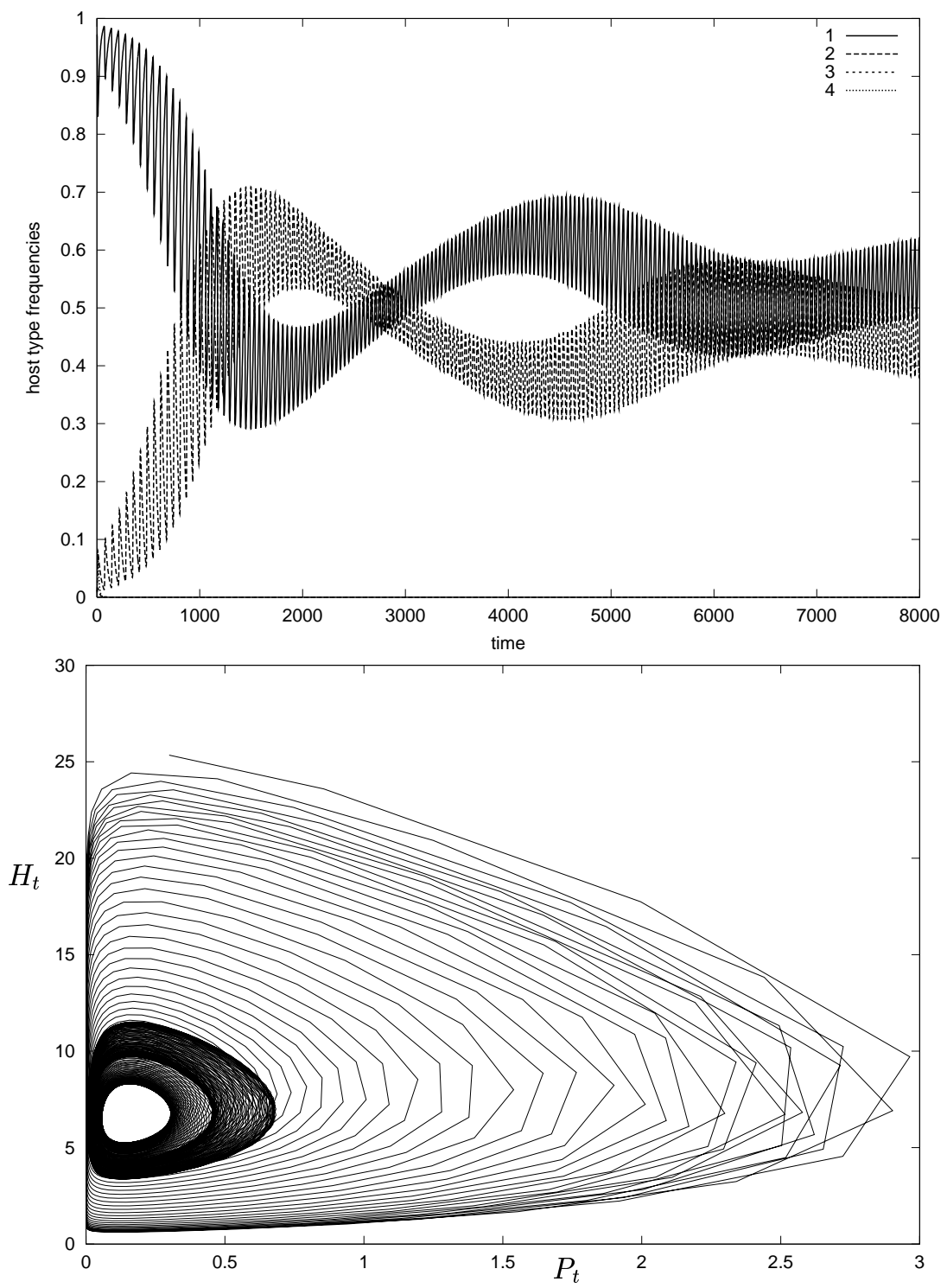

Figure 5: Numerical simulation showing quasi-periodic behaviour of both all-acceptor and egg-rejector host types (curves 1 and 2 respectively). The temporal dynamics of the host frequencies are illustrated in the top picture; the cuckoo and host densities are compared in the lower one. Parameter values and initial conditions are identical to figure 2, with the exception of $k=3000$ and $s_{H}=0.378$. Curves 2 and 4 (chick rejection and all-rejection) quickly drop to zero and remain there. 


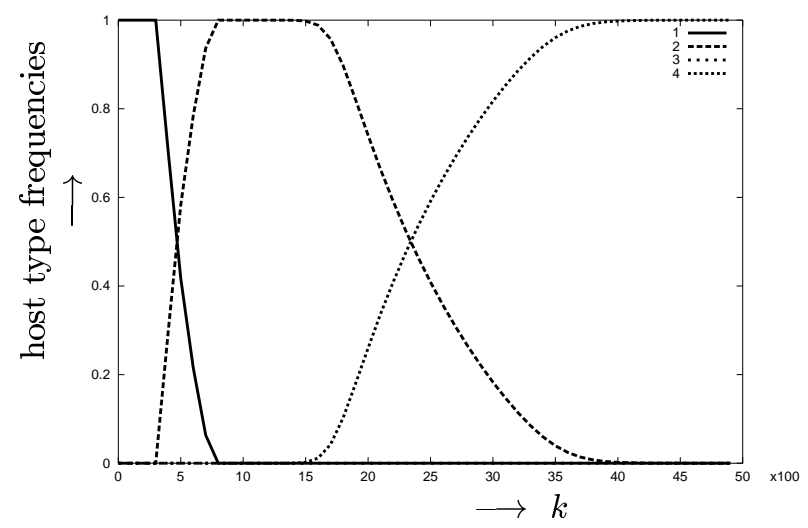

Figure 6: For small values of $k$ we find coexistence between all-acceptors (curve 1) and egg-rejectors (curve 2), but for larger values the all-rejectors (curve 4) start to increase: we find a steady state $S^{e, e c}$ in which all-rejectors can be found alongside the egg-rejectors. For even larger $k$ the all-rejectors are the only hosts. In all these cases we have $P^{e}<P^{c}, P^{e c}$. The chick-rejectors (curve 3) are absent for all $k$. Parameters used are as in figure 2, but with $f=0.71212, e_{1}=0.882$ and $c_{1}=0.934$.

denoted by $S^{e, e c}$ and is of the form

$$
S^{e, e c}=\left(P^{e, e c}, H^{e, e c}, 0, h^{e}, 0, h^{e c}\right) .
$$

Similarly, we may encounter a steady state $S^{c, e c}$ in which chick-rejectors and all-rejectors are coexisting in intermediate frequencies of the form

$$
S^{c, e c}=\left(P^{c, e c}, H^{c, e c}, 0,0, h^{c}, h^{e c}\right),
$$

or an equilibrium $S^{e, c}$,

$$
S^{e, c}=\left(P^{e, c}, H^{e, c}, 0, h^{e}, h^{c}, 0\right),
$$

in which we find egg-rejectors and chick-rejectors The analytical derivations of these equilibria are given in the Appendix.

Still other combinations of host types in equilibrium are possible, but we have only found them when two or more equilibrium cuckoo densities are chosen to be equal, e.g. $P^{e}=P^{c}<P^{e c}$. In such cases one may find one-parameter families of steady states when two cuckoo densities are the same, or a two-parameter family if $P^{e}=P^{c}=P^{e c}$. Since this is biologically implausible, these mathematical properties of the model don't provide additional insight in this biological context.

\section{DISCUSSION AND BIOLOGICAL IMPLICATIONS}

With these results at our disposal, we will now give a reflection on some current views in ecology, and more specifically on the original question: why do we not find cuckoo hosts that are able to defend themselves by recognizing an aberrant cuckoo chick in their nest?

Before giving a detailed account on the question of why chick rejection has not evolved among cuckoo hosts, we will first put the model in an ecological perspective.

\section{Predator-prey systems}

As a short remark we note that the model displays damped oscillatory behaviour, common to predatorprey systems (see e.g. (May 1973)) and illustrated in figure 2. 


\begin{tabular}{|l|c|}
\hline host species & survival rate $s_{H}$ \\
\hline Reed Warbler (Acrocephalus scirpaceus) & $0.37-0.51$ \\
Meadow Pipit (Anthus pratensis) & 0.83 \\
Wren (Troglodytes troglodytes) & $0.58-0.63$ \\
Dunnock (Prunella modularis) & 0.51 \\
Redstart (Phoenicurus phoenicurus) & $0.51-0.71$ \\
\hline
\end{tabular}

Table 1: Survival rates for various well-known host species of the cuckoo (Cramp 1988, Cramp and Brooks 1992).

\section{Diploid model}

We have also looked at a full diploid system (e.g. as in (Takasu et al. 1993, Takasu 1998)), and may conclude that although there are some quantitative differences, the overall effects of both models are qualitatively the same. The main difference is to be seen in the invasion speeds. The process of recombination slows down the increase of the selectively advantageous hosts, and vice versa for selectively disadvantageous hosts.

\section{Fitting the model to nature}

As we have seen in the analysis, the model may display a sensitive dependence on parameters. For instance, we have found a small interval in the $s_{H}$-range, where sudden changes in the qualitative behaviour take place. We briefly compare this interval with experimental values. Table 1 shows the survival rates for a number of well-known hosts of the cuckoo.

Takasu et al. (1993) have used somewhat lower values $\left(s_{H} \sim 0.2,0.5\right)$. The $s_{H}$ values in which the periodic solutions have been found are in the lower range of the experimental values. For instance, taking the parameter values of figure 5 we find oscillatory behaviour roughly in the interval $s_{H} \in(0.365,0.38)$ as shown in figure 4 . Hence we may have to be careful with the interpretation of population dynamics in species which exhibit survival rates at this lower end of the spectrum: they may display sustained defensive rejection behaviour for $s_{H}$ values even below the critical asymptotic value.

\section{Perfect defence is not sustainable}

We have discussed the existence of the equilibria $S^{e}, S^{c}$ and $S^{e c}$ in which we find coexistence of two host types, namely the acceptors and one defensive host type. In the Appendix we have given the full mathematical derivation of these solutions. At each of these three equilibria the values of the fitness functions of the respective host types are equal. This simplifies the procedure. To give an example, for $S^{e}$ we find that egg-rejection hosts have the same fitness as acceptor hosts at the given cuckoo density. In mathematical terms, and recalling that

$$
S^{e}=\left(P^{e}, H^{e}, h^{a}, h^{e}, 0,0\right)
$$

by equation (7), we have

$$
f_{a}\left(P^{e}\right)=f_{e}\left(P^{e}\right)
$$

From this expression we infer that this gives only relevant solutions provided $P^{e}>0$, or

$$
P^{e}=\frac{1}{a} \log \left(\frac{1-e_{1}+e_{2}}{e_{2}}\right)>0
$$

Hence, if we were to exclude any discrimination costs by setting $p_{e}=0$, and hence $e_{1}=1$, we would find $P^{e}=0$. Similarly, if the chick-rejectors or all-rejectors were to defend themselves perfectly, giving 
cost coefficients of $c_{1}$ and $k_{1}=1$, we would obtain $P^{c}=0$ and $P^{e c}=0$ respectively. So for the cuckoo it is crucial that the hosts display imperfect defence behaviour. Also from a physiological point of view there may be consequences. Let us suppose that the defensive hosts have to incur some physiological cost associated with the ability to discriminate eggs or chicks (a cost we have so far neglected). Now, if we introduce a perfectly-defending host type in an otherwise accepting host population, the defending hosts will increase in number, and will drive the cuckoos to extinction. From that moment on, the two host types will be selectively neutral with respect to defence behaviour, but the accepting hosts will have a selective advantage in terms of the physiological costs they don't have to incur. Heuristically, we may thus conclude that in the long run we expect to find non-defending hosts again.

In short, the assumption that hosts birds defend themselves imperfectly by making type I errors $\left(p_{e}\right.$, $\left.p_{c}>0\right)$ is a necessary condition for any discrimination behaviour to be sustained within this model.

The non-prevalence of chick rejection

We now turn to more specific considerations and formulate more precisely why, according to our findings, chick rejection is not found in nature.

Chick-rejection behaviour is incorporated in this model in two host types: the chick-rejectors and the all-rejectors. As we have seen, in most cases the heuristic argument given at the beginning of the Analysis section is applicable. Hence, to explain why we do not see chick-rejectors rather than egg-rejectors we have to show that $P^{e}<P^{c}$ for realistic parameter values. The conditions for this are

$$
\frac{1-e_{1}}{e_{2}}<\frac{1-c_{1}}{c_{2}}
$$

After some algebra this reduces to

$$
\gamma<\gamma_{e c}=\frac{p_{c}}{1-q_{c}} \frac{1-q_{e}}{p_{e}} \frac{1-b_{c}}{1-b_{e}}
$$

Now $b_{c} \leq b_{e}$, so that $\left(1-b_{c}\right) /\left(1-b_{e}\right) \geq 1$, and $\gamma$, the average fraction of the clutch saved by parasitised chick-rejectors, satisfies $\gamma<1$. The combination $p_{e} /\left(1-q_{e}\right)$ is a measure of how difficult it is to discriminate eggs. It increases with the probability of either type I or type II errors. Hence, unless it is more difficult by a sufficient margin to discriminate eggs than chicks, so that

$$
\frac{p_{e}}{1-q_{e}} \frac{1-q_{c}}{p_{c}} \geq \frac{1-b_{e}}{1-b_{c}} \frac{1}{\gamma}>1
$$

chick-rejection cannot compete with egg-rejection. If $\gamma$ is small, as it might be unless chick-rejectors keep a close watch on their nest, then chick-discrimination must be much easier than egg-discrimination for chick-rejection to prevail. It has been suggested that to spot a cuckoo chick might be more difficult than to recognize a cuckoo egg (Davies and Brooke 1988). The chicks are born within a number of days and their appearance changes quickly due to rapid growth. Eggs may look more homogeneous. This suggests that $p_{e} /\left(1-q_{e}\right) \leq p_{c} /\left(1-q_{c}\right)$, and under these circumstances egg-rejectors always outcompete chick-rejectors.

All-rejectors are fitter than egg-rejectors if they are parasitised (since $k_{2}>e_{2}$ ). To explain why we do not see all-rejectors rather than egg-rejectors we have to show that $P^{e}<P^{e c}$ for realistic parameter values. The conditions for this are

$$
\frac{1-e_{1}}{e_{2}}<\frac{1-k_{1}}{k_{2}}
$$


Defining $\alpha=e_{1}-k_{1}>0, \beta=k_{2}-e_{2}>0$, this is equivalent to

$$
\frac{1-e_{1}}{e_{2}}<\frac{\alpha}{\beta},
$$

or

$$
\gamma<\gamma_{e k}=\frac{1-b_{c}}{1-b_{e}} \frac{p_{c}}{1-q_{c}} \frac{1-q_{e}}{p_{e}} \frac{1-p_{e}}{q_{e}}\left\{1+\frac{p_{e}\left(b_{e}-b_{e c}\right)}{\left(1-p_{e}\right)\left(1-b_{c}\right)}\right\} .
$$

The term in the braces is greater than 1. If egg-rejection errors are sufficiently small that $p_{e}+q_{e}<1$, then $\left(1-p_{e}\right) / q_{e}>1$. Egg discrimination would be extremely poor if this inequality did not hold. Either $p_{e}$ or $q_{e}$ or both would be greater than $\frac{1}{2}$, meaning that the host would be more likely than not to make an error of type I, type II, or both. It follows that $\gamma_{e k}>\gamma_{e c}$, so that if the chick-rejector inequality $\gamma<\gamma_{e c}$ holds, then

$$
\gamma<\gamma_{e c}<\gamma_{e k}
$$

and the all-rejector inequality also holds. It is easier for chick-rejectors than all-rejectors to invade a steady state consisting of egg-rejectors and all-acceptors. At first sight this is a surprising result, and it is interesting to see why it holds. We may trace it back to the fact that $\beta=k_{2}-e_{2}$ is small unless errors are large. Even when all-rejectors have an advantage, which is when parasitism pressure is high, that advantage is small, only being brought into play when, through an error, the first line of defence has failed. Compounding the difficulty, the stable steady state, where the system ends up, is the one where parasitism pressure is lowest, and even this small advantage is likely to disappear.

This result may be applied much more generally. Similar arguments could be advanced for any situation where two consecutive lines of defence against parasitism or predation were possible, and we would predict that although in certain circumstances either one could prevail, the strategy of maintaining both defence systems would only be worthwhile if there was a high probability that the first one would fail.

\section{Evolutionary lag or equilibrium?}

There has been a long debate whether the observed lack of chick rejection (and also the variation in egg rejection in different host species) in avian brood parasitism is due to an evolutionary lag (Dawkins and Krebs 1979, Zahavi 1979, Lotem 1993, Soler et al. 1995) or whether the present situation is one of evolutionary equilibrium (Davies and Brooke 1988, Rothstein 1990). We will argue that the model captures both ideas, and by choosing discrimination costs appropriately, we will see that the model is in agreement with both of these views.

These hypotheses are both concerned with the explanation of one trait. If we want to view chick discrimination from the viewpoint of the lag hypothesis, we assume a lack of hereditary variation in the gene coding for this trait and sufficient benefit for the host to have the ability reject a cuckoo chick (Rothstein 1975). Hence we assume $c_{1}$ and $c_{2}$ to be large, so chick discrimination is quick $(\gamma$ close to 1$)$ and easy $\left(p_{c}\right.$ and $q_{c}$ are close to zero). There are no restrictions on $k_{1}, k_{2}, e_{1}$ or $e_{2}$. The critical point would be values of $c_{1}$ and $c_{2}$ such that

$$
\frac{1-c_{1}}{c_{2}}=\frac{1-e_{1}}{e_{2}}
$$

The model predicts that, starting with any non-trivial number of chick-rejectors, if chick rejection is the attracting steady state, the system will converge to this steady state. 
If we assume higher costs for chick-discrimination, so that it is either slow or difficult, hence assuming $c_{1}$ and $c_{2}$ to be low and thereby questioning the adaptiveness of this trait, we are in the domain of the equilibrium hypothesis. This view assumes moreover that there is sufficient hereditary variability in the host population, i.e. the number of chick-rejectors in the host population is non-trivial. Again there are no constraints on any of the other four discrimination costs. The critical point is again given by equation (10), and the model predicts that chick rejection should prevail if these discrimination costs are low enough.

\section{Further modelling and experimental work}

Finally we summarise the experimental work needed to test these results. Most important, the values of $e_{1}, c_{1}$ and $k_{1}$ (the cost due to type I errors for egg-rejectors, chick-rejectors and all-rejectors respectively), $e_{2}, c_{2}$ and $k_{2}$ (the cost due to type II errors for the egg-, chick- or all-rejector) should be assessed in the field in order to find out whether the present analysis is consistent with the biological situation. It may however prove difficult to assess $c_{1}, c_{2}, k_{1}$ and $k_{2}$ since no hosts have been found to discriminate against chicks. Knowledge of the host survival rate $s_{H}$ is required since we have found a sensitive dependence of the qualitative behaviour of the model on this parameter.

No attention has been paid in this study to the genetic variation of the cuckoo population. Since we have set out to investigate an evolutionary arms race in which one adaptation is the cause of another (Davies 2000), it is advisable to perform such an analysis in the future (Winfree 1999).

The model results have shown that the counterintuitive observations can be placed into a rational context which, if supported by additional experimental data, may resolve a long-standing question in evolutionary ecology.

\section{Acknowledgements}

RP would like to thank L.A. Peletier of the University of Leiden, the Centre for Mathematical Biology at the University of Bath and the Centrum voor Wiskunde en Informatica in Amsterdam for funding this research, and T.P. Hobson for his excellent comments on early drafts of the paper.

\section{APPENDIX}

We start the more formal mathematical investigations with an explicit formulation of the three equilibria $S^{e}, S^{c}$ and $S^{e c}$. We will do these derivations for $S^{e}$ and leave the rest of the details to the interested reader.

$S^{e}$ is converged upon if the fitness of egg-rejectors and all-acceptors is equal. By solving the fitness equations $f_{a}=f_{e}$ for $P_{t}$ we find that at $S^{e}$

$$
P_{t}=P^{e}:=\frac{1}{a} \log \left(\frac{1-e_{1}+e_{2}}{e_{2}}\right)
$$

For easy reference in the discussion, the other equilibrium values for the cuckoo for $S^{c}$ and $S^{e c}$ are given here.

$$
\begin{gathered}
P_{t}=P^{c}:=\frac{1}{a} \log \left(\frac{1-c_{1}+c_{2}}{c_{2}}\right) \\
P_{t}=P^{e c}:=\frac{1}{a} \log \left(\frac{1-k_{1}+k_{2}}{k_{2}}\right)
\end{gathered}
$$


If we introduce arbitrary (non-trivial) host type frequencies for all four host types, and the system converges to $S^{e}$, then we will see that the chick-rejectors and all-rejectors have vanished at steady state: they have been outcompeted by the fitter egg-rejectors. Hence, using eq. (11) and setting $h^{c}=h^{e c}=0$, we can solve eq. (2) at steady state to find

$$
H_{t}=H^{e}:=k\left(s_{H}+\frac{f e_{2}}{1-e_{1}+e_{2}}-1\right) .
$$

To express $h^{e}$ at steady state we introduce

$$
A(x)=\frac{\left(1-s_{P}\right) \log x}{a G\left(s_{H}+\frac{f}{x}-1\right)\left(1-\frac{1}{x}\right)} .
$$

Now we find $h^{e}$ and $h^{a}$ at steady state by solving eq. (1):

$$
\begin{array}{r}
h^{a}=1-\frac{1}{1-q_{e}}\left(1-\frac{1}{k} A\left(\frac{1-e_{1}+e_{2}}{e_{2}}\right)\right), \\
h^{e}=\frac{1}{1-q_{e}}\left(1-\frac{1}{k} A\left(\frac{1-e_{1}+e_{2}}{e_{2}}\right)\right) .
\end{array}
$$

In conclusion, we find

$$
S^{e}=\left(P^{e}, H^{e}, h^{a}, h^{e}, 0,0\right) .
$$

The derivation of the other equilibria is done in a completely analygous fashion. For chick and all rejection the expressions are given upon substitution of $c_{i}$ and $k_{i}$ for $e_{i}$ respectively, $i=1,2$, and similarly $q_{c}$ and $q_{e c}$ for $q_{e}$, in all equilibrium expressions for egg rejection. The formulae for the three other steady states - egg and all rejection, chick and all rejection and egg and chick rejection-are slightly more complicated but found in precisely the same way.

We make the following remarks, which apply for all three $S^{e}, S^{c}$ and $S^{e c}$ but to avoid iteration are only stated for $S^{e}$. The steady state $S^{e}$ is illustrated in figure 3 in terms of $h^{e}$ with respect to our chosen bifurcation parameter $k$. It intersects $h^{e}=0$ and $h^{e}=1$ in two points. In the interval between these points we hence have coexistence of egg-rejectors with all-acceptors. We extend $S^{e}$ by including the steady states $h^{e}=0$ and $h^{e}=1$, and denote this by $\overline{S^{e}}$.

A necessary condition for $S^{e}$ to be attained is $h^{e}>0$. Using this condition, we can find a minimal value for $k$ for this condition to be satisfied, denoted by $k_{0}^{e}$, such that $h^{e}>0$ :

$$
k_{0}^{e}=A\left(\frac{1-e_{1}+e_{2}}{e_{2}}\right) .
$$

Recall that $k^{P}$ is the critical value for the existence of the cuckoo population, given by $k^{P}=(1-$ $\left.s_{P}\right) / a G\left(s_{H}+f-1\right)$. Note that $k^{P}<k_{0}^{e}$. For $k$ in the interval $\left(k^{P}, k_{0}^{e}\right)$, the system converges to $S^{1}$. 
Another necessary condition for $S^{e}$ to be attained is that $h^{e}<1$. Following the same procedure, we find a maximal value for $k$, denoted by $k_{1}^{e}$,

$$
k_{1}^{e}=\frac{1}{q_{e}} A\left(\frac{1-e_{1}+e_{2}}{e_{2}}\right) .
$$

If we solve $h^{e}=0$ for $s_{H}$ we find an asymptotic value for which $S^{e}$ can exist. Denoting it by $s_{H}^{e}$, it is given by

$$
s_{H}^{e}=1-\frac{f e_{2}}{1-e_{1}+e_{2}} .
$$

An illustration of this asymptote is given in figure 4 . Under the assumption that $e_{1}, c_{1} \geq k_{1}$ and $k_{2} \geq e_{2}, c_{2}$ one can show that $s_{H}^{e c} \geq s_{H}^{e}, s_{H}^{c}$, and $k_{0}^{e c} \geq k_{0}^{e}, k_{0}^{c}$.

Dynamical behaviour of the three equilibria

With these explicit equilibrium solutions we investigate which of these equilibria is attained by the system. Suppose that for instance $P^{e}<P^{c}<P^{e c}$, that $k$ is sufficiently large, and that all host type frequencies are non-trivial. Then if we start at some $P_{0}>P^{e c}$, we know by construction of the fitness functions, that the all-rejection hosts have highest fitness (see figure 1). Hence, they will cause the cuckoo population to decrease. By the decreasing nature of $f_{e}$, there comes a point where egg rejection becomes fitter than all-rejection. This is reflected by the fact that we have assumed that $P^{e}<P^{e c}$. So the cuckoo population will decrease further and will eventually spiral around $P^{e}$ : for $P_{t}<P^{e}$ the all-acceptors are fittest and their increase allows $P_{t}$ to increase; the opposite effect is seen when $P_{t}>P^{e}$. If we assume that these oscillations converge to an equilibrium, we only have one option: the system converges to $S^{e}$. This argument indicates that the relative position of the equilibrium cuckoo populations may determine to which solution the system converges.

As we have seen in the previous paragraph, we can determine to which equilibrium solution the system converges by looking at the relative positions of $P^{e}, P^{c}$ and $P^{e c}$. It is immediate that $P^{e}<P^{c}$ if $\frac{1-e_{1}+e_{2}}{e_{2}}<\frac{1-c_{1}+c_{2}}{c_{2}}$, and analogous identities for the other options. 


\section{References}

1. D.K. Arrowsmith and C.H. Place. Introduction to Dynamical Systems. Cambridge University Press, 1990.

2. M. de L. Brooke and N.B. Davies. Provisioning of nestling cuckoos Cuculus canorus by reed warbler Acrocephalus scirpaceus hosts. Ibis, 131:630-632, 1989.

3. L.C. Brooker, M.G. Brooker, and A.M.H. Brooker. An alternative population/genetic model for the evolution of egg mimesis, and egg crypsis in cuckoos. J. Theor. Biol., 146:123-143, 1990.

4. S. Cramp. Handbook of the birds of Europe, the Middle East and North Africa, volume V. Oxford University Press, New York, 1988.

5. S. Cramp and D.J. Brooks. Handbook of the birds of Europe, the Middle East and North Africa, volume VI. Oxford University Press, New York, 1992.

6. N.B. Davies. Cuckoos, cowbirds and other cheats. T. \& A.D. Poyser, London, 2000.

7. N.B. Davies and M. de L. Brooke. Cuckoos versus reed warblers: adaptations and counteradaptations. Anim. Beh., 36:262-284, 1988.

8. N.B. Davies and M. de L. Brooke. An experimental study of co-evolution between the cuckoo, Cuculus canorus, and its hosts. II. host egg markings, chick discrimination and genera, discussion. J. Anim. Ecology, 58:225-236, 1989.

9. N.B. Davies, R.M. Kilner, and D.G. Noble. Nestling cuckoos Cuculus canorus exploit hosts with begging calls that mimic a brood. Proc. Roy. Soc. London B, 265:673-678, 1998.

10. R. Dawkins and J.R. Krebs. Arms races between and within species. Proc. Roy. Soc. London B, 205:489-511, 1979.

11. D.J. Futuyma. Evolutionary Biology. Sinauer Associates, Sunderland, Massachusetts, 1979.

12. L.E. Gilbert. Food web organization and the conservation of neotropical diversity. In M.E. Soulé and B.A. Wilcox, editors, Conservation Biology, pages 11-33. Sinauer Associates, Sunderland Massachusetts, 1980.

13. C. Kelly. A model to explore the rate of spread of mimicry and rejection in hypothetical populations of cuckoos and their hosts. J. Theor. Biol., 125:283-299, 1987.

14. A. Lotem. Learning to recognize nestlings is maladaptive for cuckoo Cuculus canorus hosts. 
Nature, 362:743-745, 1993.

15. K. Marchetti. Costs to defence and the persistence of parasitic cuckoos. Proc. Roy. Soc. London $B, 248: 41-45,1992$.

16. R.M. May. Stability and complexity in model ecosystems. Princeton University Press, Princeton, New Jersey, 1973.

17. R.M. May and S.K. Robinson. The population dynamics of avian brood parasitism. Am. Nat., 126:475-494, 1985.

18. A. Moksnes, E. Røskaft, and A.T. Braa. Rejection behavior by common cuckoo hosts towards artificial brood parasite eggs. Auk, 108:348-354, 1991.

19. H. Nakamura. Brood parasitism by the cuckoo Cuculus canorus in japan and the start of new parasitism on the azure-winged magpie Cyanopica cyana. Japanese J. of Orn., 39:1-18, 1990.

20. A.J. Nicholson and V.A. Bailey. The balance of animal populations, I. Proc. Zool. Soc. London, 1:551-598, 1935.

21. S. Rohwer, C.D. Spaw, and E. Røskaft. Costs to northern orioles of puncture-ejecting parasitic cowbird eggs from their nests. Auk, 106:734-738, 1989.

22. E. Røskaft, G.H. Orians, and L.D. Beletsky. Why do red-winged blackbirds accept eggs of brownheaded cowbirds? Evol. Ecol., 4:35-42, 1990.

23. I. Rothstein. Evolutionary rates and host defenses against avian brood parasitism. Am. Nat., 109:161-176, 1975.

24. S.I. Rothstein. A model system for coevolution: avian brood parasitism. Ann. Rev. Ecol. Syst., 21:481-508, 1990.

25. S.I. Rothstein. Relic behaviours, coevolution and the retention versus loss of host defences after episodes of avian brood parasitism. Anim. Behav., 61:95-107, 2000.

26. S.I. Rothstein and S.K. Robinson. Major unresolved questions in the study of avian brood parasitism. In S.I. Rothstein and S.K. Robinson, editors, Brood-parasites and their hosts, pages 419-425. Oxford University Press, 1998.

27. M. Soler, J.J. Soler, J.G. Martinez, and A.P. Møller. Chick recognition and acceptance: a weakness in magpies exploited by the parasitic great spotted cuckoo. Behav. Ecol. Sociobiol., 37:243-248, 1995.

28. F. Takasu. Why do all host species not show defense against avian brood parasitism: evolutionary lag or equilibrium? Am. Nat., 151:193-205, 1998.

29. F. Takasu, K. Kawasaki, H. Nakamura, J.E. Cohen, and N. Shigesada. Modeling the population dynamics of a cuckoo-host association and the evolution of host defences. Am. Nat., 142:819-839, 1993.

30. R. Winfree. Cuckoos, cowbirds and the persistence of brood parasitism. Trends Ecol. Evol., 14:338-343, 1999.

31. A. Zahavi. Parasitism and nest predation. Am. Nat., 113:157-159, 1979. 